\title{
AN INTEGRAL REPRESENTATION THEOREM FOR LOWER SEMICONTINUOUS ENVELOPES OF INTEGRAL FUNCTIONALS
}

\author{
ZHIPING LI \\ SCHOOL OF MATHEMATICAL SCIENCE, PEKING UNIVERSITY, \\ BEIJING 100871, P.R.CHINA
}

\begin{abstract}
In this paper, an integral representation theorem on sequentially weakly lower semicontinuous envelope of multiple integral functionals is proved for integrands which satisfy growth conditions of order $p$ and have lower compactness property. The result generalizes the standard results in the area.
\end{abstract}

\section{INTRODUCTION}

In calculus of variations, the problem of minimizing an integral functional

$$
F(u ; \Omega)=\int_{\Omega} f(x, u(x), D u(x)) d x,
$$

on a set of admissible functions

$$
\mathbb{A}=\left\{u \in W^{1, p}\left(\Omega ; R^{m}\right): u=u_{0} \text { on } \partial \Omega\right\},
$$

where $\Omega \subset R^{n}$ is a bounded open set with Lipschitz continuous boundary $\partial \Omega$ and $1 \leq p<\infty$, can often be replaced by the relaxed problem of minimizing the relaxed functional

$$
\hat{F}(u ; \Omega)=\int_{\Omega} \hat{f}(x, u(x), D u(x)) d x,
$$

on $\mathbb{A}$, where $\hat{f}(x, s, \cdot)$ is the quasiconvex envelope of $f(x, s, \cdot)$, i.e. the greatest quasiconvex function less than or equal to $f(x, s, \cdot)$ (see $[1,2,3])$. In fact, the solutions to the two problems coincide whenever $\hat{F}(\cdot, \Omega)$ happens to be the sequentially weakly lower semicontinuous envelope of $F(\cdot, \Omega)$, i.e. the

1991 Mathematics Subject Classification. 49J45, 49M20.

Key words and phrases. quasiconvex envelope, lower semicontinuous envelope, integral representation, lower compactness property.

The research was supported by the National Natural Science Foundation of China. 
greatest sequentially weakly lower semicontinuous functional defined on $\mathbb{A}$ less than or equal to $F(\cdot, \Omega)$, which is identified by (see $[3,4,5]$ )

$$
\Gamma^{-}-\lim F(u ; \Omega)=\min \left\{\liminf _{\alpha \rightarrow \infty} F\left(u_{\alpha} ; \Omega\right): u_{\alpha} \in \mathbb{A}, u_{\alpha} \rightarrow u \text { in } W^{1, p}\left(\Omega ; R^{m}\right)\right\},
$$

where "- means "converges weakly to". This is useful in numerical analysis and computations (see for example $[6,7,8,9]$ ) as well as theoretically important. Thus, it is naturally desirable to know under what conditions this is true. The following well known result is given by Acerbi and Fusco [3].

Theorem 1.1. Let $f: \Omega \times R^{m} \times R^{n \times m} \rightarrow R$ be a Carathéodory function which satisfies the hypotheses

(H1): $0 \leq f(x, s, \xi) \leq a(x)+C\left(|s|^{p}+|\xi|^{p}\right)$ for every $x \in \Omega, s \in R^{m}$, $\xi \in R^{n \times m}$, where $C$ is a nonnegative constant and $a(x) \in L^{1}(\Omega)$ is a nonnegative function;

(H2): $\left|f\left(x, s_{1}, \xi\right)-f\left(x, s_{2}, \xi\right)\right| \leq \omega\left(x,\left|s_{1}-s_{2}\right|\right) \beta(|\xi|)$, where $\omega: \Omega \times R \rightarrow$ $R^{+}$is a Carathéodory function, $\omega(x, 0)=0$, and $\beta(\cdot)$ is increasing and nonnegative.

Then, for every open subset $\Omega^{\prime} \subset \Omega, \hat{F}\left(\cdot ; \Omega^{\prime}\right)$ is the sequentially weakly lower semicontinuous envelope of $F\left(\cdot ; \Omega^{\prime}\right)$ in $W^{1, p}\left(\Omega^{\prime} ; R^{m}\right)$.

The main result of this paper (Theorem 3.1) generalizes theorem 1.1 by replacing the hypothesis (H1) by two weaker hypotheses :

(H1a): $|f(x, s, \xi)| \leq a(x)+C\left(|s|^{p}+|\xi|^{p}\right)$ for every $x \in \Omega, s \in R^{m}$, $\xi \in R^{n \times m}$, where $C$ is a nonnegative constant and $a(x) \in L^{1}(\Omega)$ is a nonnegative function;

(H1b): $f$ has the lower compactness property, i.e. $f^{-}\left(x, u_{\alpha}(x), D u_{\alpha}(x)\right)$ is precompact in $L^{1}\left(\Omega^{\prime}\right)$ whenever $\Omega^{\prime}$ is an open subset of $\Omega, u, u_{\alpha} \in$ $W^{1, p}\left(\Omega^{\prime} ; R^{m}\right)$ are such that $u_{\alpha} \rightarrow u$ in $W^{1, p}\left(\Omega^{\prime} ; R^{m}\right)$ and $F\left(u_{\alpha} ; \Omega^{\prime}\right) \leq$ $\hat{C}<+\infty$, where $f^{-}=\min \{f, 0\}$.

The proof of the main result makes fully use of theorem 1.1 and the results developed in section 2, where it is shown under the hypothesis (H1a) that

$$
\hat{f}(x, s, \xi)=\lim _{\beta \rightarrow \infty} \hat{f}_{\beta}(x, s, \xi),
$$

for all $(x, s, \xi) \in \Omega \times R^{m} \times R^{n \times m}$ and

$$
\hat{F}\left(u ; \Omega^{\prime}\right)=\lim _{\beta \rightarrow \infty} \hat{F}_{\beta}\left(u ; \Omega^{\prime}\right),
$$


for every open subset $\Omega^{\prime} \subset \Omega$ and for all $u \in W^{1, p}\left(\Omega^{\prime} ; R^{m}\right)$, where $\hat{f}_{\beta}$ is the quasiconvex envelope of

$$
f_{\beta}(x, s, \xi)=\max \{f(x, s, \xi),-\beta\}
$$

and

$$
\hat{F}_{\beta}\left(u ; \Omega^{\prime}\right)=\int_{\Omega^{\prime}} \hat{f}_{\beta}(x, u(x), D u(x)) d x .
$$

As a by-product, we obtain a theorem on lower semicontinuity of integral functionals (Theorem 3.2) which is not covered by the more general results of the kind given recently by $\mathrm{Li}[10,11]$.

Remark 1.1. Here and throughout this paper, assumptions and statements are referred to sets with measure-negligible projections on $\Omega$, i.e. they hold on a subset $\Omega^{\prime} \subset \Omega$ with meas $\left(\Omega^{\prime}\right)=\operatorname{meas}(\Omega)$ where meas $(\cdot)$ denotes the Lebesgue measure in $R^{n}$.

\section{Quasiconvex EnVElope of $f(x, s, \cdot)$}

Let $\Omega \subset R^{n}$ be a bounded open set with Lipschitz continuous boundary $\partial \Omega$ and let $1 \leq p<\infty$. Let $f: \Omega \times R^{m} \times R^{n \times m} \rightarrow R$ be a Carathéodory function. Define

$$
f_{\beta}=\max \{f,-\beta\}, \quad \forall \beta \in \mathbb{N}=\{1,2,3, \ldots\} .
$$

Denote the quasiconvex envelopes of $f_{\beta}$ and $f$ by $\hat{f}_{\beta}$ and $\hat{f}$ respectively.

Lemma 2.1. Let $f: \Omega \times R^{m} \times R^{n \times m} \rightarrow R$ be a Carathéodory function which satisfies (H1a). Then, $\left\{\hat{f}_{\beta}\right\}_{\beta=1}^{\infty}$ is a nonincreasing sequence of Carathéodory functions which satisfy

$$
-\beta \leq \hat{f}_{\beta}(x, s, \xi) \leq a(x)+C\left(|s|^{p}+|\xi|^{p}\right) \quad \forall(x, s, \xi) \in \Omega \times R^{m} \times R^{n \times m},
$$

where, as given in (H1a), $C$ is a nonnegative constant and $a(x) \in L^{1}(\Omega)$ is a nonnegative function.

Proof. By the inequality,

$\hat{f}_{\beta}(x, s, \xi) \leq f_{\beta}(x, s, \xi) \leq f_{\alpha}(x, s, \xi) \quad \forall(x, s, \xi) \in \Omega \times R^{m} \times R^{n \times m}$ and $\forall \beta \geq \alpha$,

which follows directly form the definitions, we conclude that $\hat{f}_{\beta} \leq \hat{f}_{\alpha}$ for all $\beta \geq \alpha$. That is $\left\{\hat{f}_{\beta}\right\}_{\beta=1}^{\infty}$ is a nonincreasing sequence of functions.

Next, let

$$
g_{\beta}(x, s, \xi)=f_{\beta}(x, s, \xi)+\beta
$$


Then, $\left\{g_{\beta}\right\}_{\beta=1}^{\infty}$ are Carathéodory functions satisfying (H1). As a consequence (see $[3,4,5]$ ), for each $\beta \in \mathbb{N}, \hat{g}_{\beta}$, the quasiconvex envelope of $g_{\beta}$, is a Carathéodory function and satisfies

$$
0 \leq \hat{g}_{\beta}(x, s, \xi) \leq a(x)+C\left(|s|^{p}+|\xi|^{p}\right)+\beta .
$$

Since $\hat{f}_{\beta}=\hat{g}_{\beta}-\beta,(2.2)$ follows.

Lemma 2.2. Let $f: \Omega \times R^{m} \times R^{n \times m} \rightarrow R$ be a Carathéodory function which satisfies (H1a). Then, for every $(x, s, \xi) \in \Omega \times R^{m} \times R^{n \times m}$,

$$
\bar{f}(x, s, \xi)>-\infty,
$$

where

$$
\begin{aligned}
\bar{f}(x, s, \xi)=\inf \left\{\liminf _{\alpha \rightarrow \infty} \frac{1}{\operatorname{meas}(\Omega)} \int_{\Omega} f\left(x, s, \xi+D \varphi_{\alpha}\left(x^{\prime}\right)\right) d x^{\prime}:\right. \\
\left.\varphi_{\alpha} \rightarrow 0 \text { in } W^{1, p}\left(\Omega ; R^{m}\right)\right\} .
\end{aligned}
$$

Proof. By the definition of $\bar{f}(x, s, \xi)$, there exists a sequence of functions $\left\{\varphi_{\alpha}\right\}_{\alpha=1}^{\infty} \subset W^{1, p}\left(\Omega ; R^{m}\right)$ such that

$$
\begin{gathered}
\varphi_{\alpha} \rightarrow 0 \quad \text { in } W^{1, p}\left(\Omega ; R^{m}\right), \\
\bar{f}(x, s, \xi)=\lim _{\alpha \rightarrow \infty} \frac{1}{\operatorname{meas}(\Omega)} \int_{\Omega} f\left(x, s, \xi+D \varphi_{\alpha}\left(x^{\prime}\right)\right) d x^{\prime} .
\end{gathered}
$$

It follows from (H1a) that

$$
f\left(x, s, \xi+D \varphi_{\alpha}\left(x^{\prime}\right)\right) \geq-\left[a(x)+C\left(|s|^{p}+\left|\xi+D \varphi_{\alpha}\left(x^{\prime}\right)\right|^{p}\right)\right] .
$$

This gives

$$
\begin{aligned}
& \int_{\Omega} f\left(x, s, \xi+D \varphi_{\alpha}\left(x^{\prime}\right)\right) d x^{\prime} \\
\geq & -\left[a(x)+C\left(|s|^{p}+2^{p-1}|\xi|^{p}\right)\right] \operatorname{meas}(\Omega)-2^{p-1} \int_{\Omega}\left|D \varphi_{\alpha}\left(x^{\prime}\right)\right|^{p} d x^{\prime} .
\end{aligned}
$$

Combining (2.4), (2.5) and (2.6), we obtain (2.3).

Lemma 2.3. Let $f: \Omega \times R^{m} \times R^{n \times m} \rightarrow R$ be a Carathéodory function which satisfies (H1a). Then, for every $(x, s, \xi) \in \Omega \times R^{m} \times R^{n \times m}$ the function $\tilde{f}$ defined by $\tilde{f}(x, s, \xi)=\lim _{\beta \rightarrow \infty} \hat{f}_{\beta}(x, s, \xi)$ satisfies

$$
-\infty<\tilde{f}(x, s, \xi) \leq a(x)+C\left(|s|^{p}+|\xi|^{p}\right) .
$$


Proof. Since, by lemma $2.2,\left\{\hat{f}_{\beta}\right\}$ is a nonincreasing sequence satisfying (2.2), it only remains to show that, for every $(x, s, \xi) \in \Omega \times R^{m} \times R^{n \times m},\left\{\hat{f}_{\beta}(x, s, \xi)\right\}$ is bounded from below.

Fix $(x, s, \xi) \in \Omega \times R^{m} \times R^{n \times m}$, denote $g_{\beta}(D \varphi)=f_{\beta}(x, s, \xi+D \varphi)+\beta$. Then $g_{\beta}, \beta \in \mathbb{N}$, satisfy (H1) and (H2) and $\hat{g}_{\beta}=\hat{f}_{\beta}+\beta$. Recall that the sequentially weakly lower semicontinuous envelope of $G_{\beta}(\varphi)=\int_{\Omega} g_{\beta}\left(x, s, \xi+D \varphi\left(x^{\prime}\right)\right) d x^{\prime}$ in $W^{1, p}\left(\Omega ; R^{m}\right)$ is (see $\left.[4,5]\right)$

$$
\begin{aligned}
\hat{I}_{\beta}(\varphi) & =\Gamma^{-}-\lim G_{\beta}(\varphi) \\
& =\min \left\{\liminf _{\alpha \rightarrow \infty} G_{\beta}\left(\varphi_{\alpha}\right): \varphi_{\alpha} \rightarrow \varphi, \text { in } W^{1, p}\left(\Omega ; R^{m}\right)\right\} .
\end{aligned}
$$

By lemma 2.1, (2.8) and theorem 1.1, there exists a sequence $\varphi_{\alpha} \in W^{1, p}\left(\Omega ; R^{m}\right)$ such that

$$
\begin{gathered}
\varphi_{\alpha} \rightarrow 0 \quad \text { in } W^{1, p}\left(\Omega ; R^{m}\right), \\
\hat{g}_{\beta}(0) \operatorname{meas}(\Omega)=\hat{I}_{\beta}(0)=\lim _{\alpha \rightarrow \infty} \int_{\Omega} g_{\beta}\left(D \varphi_{\alpha}\left(x^{\prime}\right)\right) d x^{\prime} .
\end{gathered}
$$

Since

$$
\begin{aligned}
\int_{\Omega} g_{\beta}\left(D \varphi_{\alpha}\left(x^{\prime}\right)\right) d x^{\prime} & =\int_{\Omega}\left(f_{\beta}\left(x, s, \xi+D \varphi_{\alpha}\left(x^{\prime}\right)\right)+\beta\right) d x^{\prime} \\
& \geq \int_{\Omega} f\left(x, s, \xi+D \varphi_{\alpha}\left(x^{\prime}\right)\right) d x^{\prime}+\beta \operatorname{meas}(\Omega)
\end{aligned}
$$

by $(2.9),(2.10)$ and lemma 2.2 , we have

$$
\hat{f}_{\beta}(x, s, \xi)=\left(\hat{g}_{\beta}(0)-\beta\right) \geq \bar{f}(x, s, \xi)>-\infty \quad \forall \beta \in \mathbb{N},
$$

where $\bar{f}(x, s, \xi)$ is defined by (2.3). This completes the proof.

Theorem 2.1. Let $f: \Omega \times R^{m} \times R^{n \times m} \rightarrow R$ be a Carathéodory function which satisfies (H1a). Then

$$
\hat{f}(x, s, \xi)=\lim _{\beta \rightarrow \infty} \hat{f}_{\beta}(x, s, \xi) \quad \forall(x, s, \xi) \in \Omega \times R^{m} \times R^{n \times m},
$$

and

$$
\int_{\Omega^{\prime}} \hat{f}(x, u(x), v(x)) d x=\lim _{\beta \rightarrow \infty} \int_{\Omega^{\prime}} \hat{f}_{\beta}(x, u(x), v(x)) d x
$$

for every measurable subset $\Omega^{\prime} \subset \Omega, u \in L^{p}\left(\Omega^{\prime} ; R^{m}\right)$ and $v \in L^{p}\left(\Omega^{\prime} ; R^{n \times m}\right)$. 
Proof. By lemma 2.3, $\tilde{f}(x, s, \xi)=\lim _{\beta \rightarrow \infty} \hat{f}_{\beta}(x, s, \xi)$ satisfies (2.7). We are going to show that $\tilde{f}(x, s, \cdot)$ is the quasiconvex envelope of $f(x, s, \cdot)$.

For fixed $(x, s, \xi) \in \Omega \times R^{m} \times R^{n \times m}$, by lemma 2.1 and the quasiconvexity of $\hat{f}_{\beta}(x, s, \xi)$ respect to $\xi($ see $[1,2,3])$,

$$
\begin{aligned}
\tilde{f}(x, s, \xi) & \leq \hat{f}_{\beta}(x, s, \xi) \\
& \leq \frac{1}{\operatorname{meas}(\Omega)} \int_{\Omega} \hat{f}_{\beta}\left(x, s, \xi+D \varphi\left(x^{\prime}\right)\right) d x^{\prime} \quad \forall \varphi \in C_{0}^{\infty}(\Omega) .
\end{aligned}
$$

Let

$$
g_{\beta}\left(x^{\prime}\right)=a(x)+C\left(|s|^{p}+\left|\xi+D \varphi\left(x^{\prime}\right)\right|^{p}\right)-\hat{f}_{\beta}\left(x, s, \xi+D \varphi\left(x^{\prime}\right)\right) .
$$

By lemma 2.1, $\left\{g_{\beta}\right\}$ is a nondecreasing sequence of nonnegative functions. By lemma 2.3,

$$
\lim _{\beta \rightarrow \infty} g_{\beta}\left(x^{\prime}\right)=g\left(x^{\prime}\right)
$$

with

$$
g\left(x^{\prime}\right)=a(x)+C\left(|s|^{p}+\left|\xi+D \varphi\left(x^{\prime}\right)\right|^{p}\right)-\tilde{f}\left(x, s, \xi+D \varphi\left(x^{\prime}\right)\right) .
$$

Hence, by Beppo Levi's theorem [12, 13], we have

$$
\int_{\Omega} g\left(x^{\prime}\right) d x^{\prime}=\lim _{\beta \rightarrow \infty} \int_{\Omega} g_{\beta}\left(x^{\prime}\right) d x^{\prime} .
$$

It follows from (2.14) and (2.15) that

$$
\tilde{f}(x, s, \xi) \leq \frac{1}{\operatorname{meas}(\Omega)} \int_{\Omega} \tilde{f}\left(x, s, \xi+D \varphi\left(x^{\prime}\right)\right) d x^{\prime} \quad \forall \varphi \in C_{0}^{\infty}(\Omega) .
$$

This implies that $\tilde{f}(x, s, \xi)$ is quasiconvex respect to $\xi$ (see $[1,2,3])$. Thus, it follows from

$$
\tilde{f}(x, s, \xi) \leq \hat{f}_{\beta}(x, s, \xi) \leq f_{\beta}(x, s, \xi), \quad \forall \beta \in \mathbb{N}
$$

and hence $\tilde{f}(x, s, \xi) \leq f(x, s, \xi)$ that

$$
\tilde{f}(x, s, \xi) \leq \hat{f}(x, s, \xi),
$$

since $\hat{f}(x, s, \cdot)$ is the greatest quasiconvex function less than or equal to $f(x, s$, .). On the other hand, since

$$
\hat{f}(x, s, \xi) \leq f(x, s, \xi) \leq f_{\beta}(x, s, \xi), \quad \forall \beta \in \mathbb{N},
$$


we have $\hat{f}(x, s, \xi) \leq \hat{f}_{\beta}(x, s, \xi)$ for all $\beta$ and hence, by (2.7)

$$
\hat{f}(x, s, \xi) \leq \tilde{f}(x, s, \xi) .
$$

It follows from (2.17) and (2.18) that

$$
\hat{f}(x, s, \xi)=\tilde{f}(x, s, \xi) \quad \forall(x, s, \xi) \in \Omega \times R^{m} \times R^{n \times m} .
$$

This proves (2.12).

Applying Beppo Levi's theorem $[12,13]$ to the sequence

$$
g_{\beta}(x)=a(x)+C\left(|u(x)|^{p}+|v(x)|^{p}\right)-\hat{f}_{\beta}(x, u(x), v(x)), \quad \beta \in \mathbb{N},
$$

which, by lemma 2.1, is nonnegative and nondecreasing and which, by (2.12), converges to

$$
g(x)=a(x)+C\left(|u(x)|^{p}+|v(x)|^{p}\right)-\hat{f}(x, u(x), v(x))
$$

as $\beta \rightarrow \infty$ for all $x \in \Omega^{\prime}$, we obtain (2.13).

Corollary 2.1. Let $f: \Omega \times R^{m} \times R^{n \times m} \rightarrow R$ be a Carathéodory function which satisfies (H1a). Then

$$
\hat{F}\left(u ; \Omega^{\prime}\right)=\lim _{\beta \rightarrow \infty} \hat{F}_{\beta}\left(u ; \Omega^{\prime}\right)
$$

for every open subset $\Omega^{\prime} \subset \Omega$ and for all $u \in W^{1, p}\left(\Omega^{\prime} ; R^{m}\right)$.

Proof. The conclusion follows directly from (2.13).

\section{Integral REPRESENTATiOn OF $\Gamma^{-}-\lim F(u ; \Omega)$}

In section 2, the relationship between the quasiconvex envelopes of $f$ and $f_{\beta}$ is discussed. In this section, we will see how the sequentially weakly lower semicontinuous envelope of $F\left(\cdot ; \Omega^{\prime}\right)$ relates to those of $F_{\beta}\left(\cdot ; \Omega^{\prime}\right)$. First, recall that they are defined by (see $[4,5])$

$$
\begin{aligned}
& \Gamma^{-}-\lim F\left(u ; \Omega^{\prime}\right)=\min \left\{\liminf _{\alpha \rightarrow \infty} F\left(u_{\alpha} ; \Omega^{\prime}\right): u_{\alpha} \rightarrow u \text { in } W^{1, p}\left(\Omega^{\prime} ; R^{m}\right)\right\}, \\
& \Gamma^{-}-\lim F_{\beta}\left(u ; \Omega^{\prime}\right)=\min \left\{\liminf _{\alpha \rightarrow \infty} F_{\beta}\left(u_{\alpha} ; \Omega^{\prime}\right): u_{\alpha} \rightarrow u \text { in } W^{1, p}\left(\Omega^{\prime} ; R^{m}\right)\right\} .
\end{aligned}
$$

Lemma 3.1. Let $f: \Omega \times R^{m} \times R^{n \times m} \rightarrow R$ be a Carathéodory function which satisfies (H1a) and (H2). Then

$$
\Gamma^{-}-\lim F_{\beta}\left(u ; \Omega^{\prime}\right)=\int_{\Omega^{\prime}} \hat{f}_{\beta}(x, u(x), D u(x)) d x
$$


for each $\beta \in \mathbb{N}$ and every open subset $\Omega^{\prime} \subset \Omega$.

Proof. For each $\beta \in \mathbb{N}$, let $g_{\beta}=f_{\beta}+\beta$. It is easily seen that $g_{\beta}$ is a Carathéodory function satisfying (H1) and (H2). Thus, by theorem 1.1,

$$
\Gamma^{-}-\lim G_{\beta}\left(u ; \Omega^{\prime}\right)=\int_{\Omega^{\prime}} \hat{g}_{\beta}(x, u(x), D u(x)) d x
$$

for every open subset $\Omega^{\prime} \subset \Omega$, where

$$
G_{\beta}\left(u ; \Omega^{\prime}\right)=\int_{\Omega^{\prime}} g_{\beta}(x, u(x), D u(x)) d x
$$

and $\hat{g}_{\beta}(x, s, \cdot)$ is the quasiconvex envelope of $g_{\beta}(x, s, \cdot)$. Since $($ see $[4,5])$

$$
\begin{gathered}
\Gamma^{-}-\lim F_{\beta}\left(u ; \Omega^{\prime}\right)=\Gamma^{-}-\lim G_{\beta}\left(u ; \Omega^{\prime}\right)-\beta \operatorname{meas}\left(\Omega^{\prime}\right), \\
\hat{f}_{\beta}(x, s, \xi)=\hat{g}_{\beta}(x, s, \xi)-\beta
\end{gathered}
$$

(3.3) follows from (3.4).

Lemma 3.2. Let $f: \Omega \times R^{m} \times R^{n \times m} \rightarrow R$ be a Carathéodory function which satisfies (H1a), (H1b) and (H2). Then

$$
\Gamma^{-}-\lim F\left(u ; \Omega^{\prime}\right)=\lim _{\beta \rightarrow \infty} \Gamma^{-}-\lim F_{\beta}\left(u ; \Omega^{\prime}\right)
$$

for every open subset $\Omega^{\prime} \subset \Omega$ and $u \in W^{1, p}\left(\Omega^{\prime} ; R^{m}\right)$.

Proof. Let an open subset $\Omega^{\prime} \subset \Omega$ and a function $u \in W^{1, p}\left(\Omega^{\prime} ; R^{m}\right)$ be given. It follows from lemma 2.1 and lemma 3.1 that $\Gamma^{-}-\lim F_{\beta}\left(u ; \Omega^{\prime}\right), \beta \in \mathbb{N}$, are nonincreasing. Since obviously $\Gamma^{-}-\lim F\left(u ; \Omega^{\prime}\right) \leq \Gamma^{-}-\lim F_{\beta}\left(u ; \Omega^{\prime}\right)$ for every $\beta \in \mathbb{N}$, we have

$$
\Gamma^{-}-\lim F\left(u ; \Omega^{\prime}\right) \leq \lim _{\beta \rightarrow \infty} \Gamma^{-}-\lim F_{\beta}\left(u ; \Omega^{\prime}\right)
$$

To show the inverse inequality, let $\left\{u_{\alpha}\right\}_{\alpha=1}^{\infty} \subset W^{1, p}\left(\Omega^{\prime} ; R^{m}\right)$ be such that (see $(3.1)$

$$
\begin{gathered}
u_{\alpha} \rightarrow u \quad \text { in } W^{1, p}\left(\Omega^{\prime} ; R^{m}\right), \\
\Gamma^{-}-\lim F\left(u ; \Omega^{\prime}\right)=\lim _{\alpha \rightarrow \infty} F\left(u_{\alpha} ; \Omega^{\prime}\right) .
\end{gathered}
$$

For any $\epsilon>0$, by (H1b), (3.7) and (3.8), there exists $\delta(\epsilon)>0$ such that

$$
\left|\int_{E} f^{-}\left(x, u_{\alpha}(x), D u_{\alpha}(x)\right) d x\right|<\epsilon \quad \forall \alpha \in \mathbb{N}
$$


whenever $E \subset \Omega^{\prime}$ is measurable and meas $(E)<\delta(\epsilon)$. Denote

$$
\Omega_{\beta, \alpha}^{\prime}=\left\{x \in \Omega^{\prime}: f\left(x, u_{\alpha}(x), D u_{\alpha}(x)\right) \leq-\beta\right\} .
$$

Then, (3.9) implies that

$$
\operatorname{meas}\left(\Omega_{\beta, \alpha}^{\prime}\right) \rightarrow 0 \quad \text { uniformly for all } \alpha \text { as } \beta \rightarrow \infty \text {. }
$$

This in turn implies that for any $\epsilon>0$ there exists $\beta(\epsilon)>0$ such that

$$
\left|\int_{\Omega^{\prime} \beta, \alpha} f^{-}\left(x, u_{\alpha}(x), D u_{\alpha}(x)\right) d x\right|<\epsilon \quad \forall \alpha \in \mathbb{N} \text { and } \beta>\beta(\epsilon) .
$$

Since

$$
\begin{aligned}
F\left(u_{\alpha} ; \Omega^{\prime}\right) & =F_{\beta}\left(u_{\alpha} ; \Omega^{\prime}\right)+\left(F\left(u_{\alpha} ; \Omega^{\prime}\right)-F_{\beta}\left(u_{\alpha} ; \Omega^{\prime}\right)\right) \\
& \geq F_{\beta}\left(u_{\alpha} ; \Omega^{\prime}\right)+\int_{\Omega^{\prime}{ }_{\beta, \alpha}} f^{-}\left(x, u_{\alpha}(x), D u_{\alpha}(x)\right) d x \quad \forall \alpha, \beta \in \mathbb{N},
\end{aligned}
$$

by (3.8) and (3.10),

$$
\Gamma^{-}-\lim F\left(u ; \Omega^{\prime}\right) \geq \Gamma^{-}-\lim F_{\beta}\left(u ; \Omega^{\prime}\right)-\epsilon \quad \forall \beta>\beta(\epsilon),
$$

and hence, by the arbitrariness of $\epsilon>0$, we conclude

$$
\Gamma^{-}-\lim F\left(u ; \Omega^{\prime}\right) \geq \lim _{\beta \rightarrow \infty} \Gamma^{-}-\lim F_{\beta}\left(u ; \Omega^{\prime}\right) .
$$

This completes the proof.

Theorem 3.1. Let $f: \Omega \times R^{m} \times R^{n \times m} \rightarrow R$ be a Carathéodory function which satisfies (H1a), (H1b) and (H2). Then, for every open subset $\Omega^{\prime} \subset \Omega$,

$$
\hat{F}\left(u ; \Omega^{\prime}\right)=\Gamma^{-}-\lim F\left(u ; \Omega^{\prime}\right) \quad \forall u \in W^{1, p}\left(\Omega^{\prime} ; R^{m}\right) .
$$

In other words, $\hat{F}\left(\cdot ; \Omega^{\prime}\right)$ is the sequentially weakly lower semicontinuous envelope of $F\left(\cdot ; \Omega^{\prime}\right)$ in $W^{1, p}\left(\Omega^{\prime} ; R^{m}\right)$.

Proof. The theorem follows from theorem 2.1, lemma 3.1 and lemma 3.2 by combing (2.13), (3.3) and (3.5).

As a corollary of theorem 3.1, we have

Theorem 3.2. Let $f: \Omega \times R^{m} \times R^{n \times m} \rightarrow R$ be a Carathéodory function which satisfies (H1a), (H1b) and (H2). Let $u_{\alpha}, u \in W^{1, p}\left(\Omega^{\prime} ; R^{m}\right)$ be such that

$$
u_{\alpha} \rightarrow u \quad \text { in } W^{1, p}\left(\Omega^{\prime} ; R^{m}\right) .
$$


Then

$$
\int_{\Omega} \hat{f}(x, u(x), D u(x)) d x \leq \liminf _{\alpha \rightarrow \infty} \int_{\Omega} \hat{f}\left(x, u_{\alpha}(x), D u_{\alpha}(x)\right) d x .
$$

That is the functional $\hat{F}(\cdot ; \Omega)$ is sequentially weakly lower semicontinuous on $W^{1, p}\left(\Omega ; R^{m}\right)$.

\section{REFERENCES}

[1] C. B. Morrey, Multiple Integrals in the Calculus of Variations. Springer, New York, 1966.

[2] J. M. Ball and F. Murat $W^{1, p}$-quasiconvexity and variational problems for multiple integrals. J. Funct. Anal., 58, (1984)225-253.

[3] E. Acerbi and N. Fusco, Semicontinuity problems in the calculus of variations. Arch. Rat. Mech. Anal. 86(1984), 125-145.

[4] B. Dacorogna, Direct Methods in the Calculus of Variations, Applied Mathematical Sciences, 78, Springer-Verlag, Berlin, 1989.

[5] G. Buttazzo, Semicontinuity, Relaxation and Integral Representation in the Calculus of Variations. Pitman Research Notes in Mathematics Series 207. Longman, 1989.

[6] M. Chipot, Numerical analysis of oscillations in nonconvex problems. Numer. Math., 59 (1991), 747-767.

[7] C. Collins, D. Kinderlehrer and M. Luskin, Numerical approximation of the solution of a variational problem with a double well potential. SIAM J. Numer. Anal., 28(1991), 321-332.

[8] Z.-P. Li, Computation of microstructures by using quasiconvex envelope. Research Report No.61, Institute and School of Mathematics, Peking University, 1995.

[9] Z.-P. Li, Simultaneous numerical approximation of microstructures and relaxed minimizers. To appear Numer. Math..

[10] Z.-P. Li, Lower semicontinuity of multiple integrals and convergent integrands. ESAIM: Control, Optimisation and Calculus of Variations, 1(1996), 169-189.

[11] Z.-P. Li, A theorem on lower semicontinuity of integral functionals. Proc. Roy. Soc. Edinburgh, 126A(1996), 363-374.

[12] R.L. Whecdan and A. Zygmund, Measures and Integrations. Marcel Dekker, INC., New York and Basel, 1977.

[13] R.A. Adams, Sobolev Spaces. Academic Press, New York, 1975. 